\title{
$k$-Prime labeling of certain cycle connected graphs
}

\author{
S. Teresa Arockiamary ${ }^{1 *}$ and G. Vijayalakshmi ${ }^{2}$
}

\begin{abstract} paper, we investigate some cycle related graphs which admit $k$ - prime labeling.

Keywords

$k$ - prime labeling, Cycle graph, Tadpole graph, Barycentric subdivision, Friendship graph.

\section{AMS Subject Classification}

26A33, 30E25, 34A12, 34A34, 34A37, 37C25, 45J05.

${ }^{1}$ Assistant Professor, Department of Mathematics, Stella Maris College, Chennai, Tamil Nadu, India.

${ }^{2}$ Research Scholar, Department of Mathematics, Stella Maris College, Chennai, Tamil Nadu, India.

*Corresponding author: ${ }^{1}$ teresa_aroc@yahoo.co.in; ${ }^{2}$ viji.gsekar@gmail.com

Article History: Received 22 November 2018; Accepted 09 May 2019
\end{abstract}

A $k$ - prime labeling of a graph $G$ is an injective function $f: V \rightarrow\{k, k+1, k+2, \ldots, k+|V|-1\}$ for some positive integer $k$ that induces a function $f^{+}: E(G) \rightarrow N$ of the edges of $G$ defined by $f^{+}(u v)=g c d(f(u), f(v)), \forall e=u v \in$ $E(G)$ such that $\operatorname{gcd}(f(u), f(v))=1$. A graph $G$ that admits $k$-prime labeling is called a $k$ - prime graph. In this

(C)2019 MJM

\section{Contents}

1 Introduction .............................. 280

2 Preliminaries .............................280

3 Main Results ........................... 280

4 Conclusion ............................... 283

References

283

\section{Introduction}

In our study we consider simple, finite, undirected and non trivial graph $G=(V, E)$ with the vertex set $V$ and the edge set $E$. The idea of prime labeling was introduced by Entringer and the problem was discussed by A. Tout, A.N. Dabboucy and K. Howalla. [1]. Later Vaidya and Prajapati [2] studied the concept of $k$-prime labeling. They proved that $P_{m}$ is $k$ - prime for each positive integer $k$. In our work we have discussed $k$ - prime labeling for all positive integer $k \geq 2$.

\section{Preliminaries}

We begin with the definition of $k$ - prime labeling.

Definition 2.1. A $k$-prime labeling of a graph $G$ is an injective function $f: V \rightarrow\{k, k+1, k+2, \ldots, k+|V|-1\}$ for some positive integer $k$ that induces a function $f^{+}: E(G) \rightarrow N$ of the edges of $G$ defined by $f^{+}(u v)=\operatorname{gcd}(f(u), f(v)), \forall e=$ $u v \in E(G)$ such that $\operatorname{gcd}(f(u), f(v))=1$. A graph $G$ that admits $k$-prime labeling is called a $k$-prime graph.
The definition of Tadpole Graph $T_{m, n}$, Barycentric Subdivision of cycles $C_{n}$ and Friendship Graph $F_{n}$ are available in literature.

\section{Main Results}

Theorem 3.1. Even Cycle $C_{2 n}$ is $k$-prime for $n \geq 2$.

Proof. Let us denote the vertices and edges of $C_{2 n}$ as

$V=\left\{v_{1}, v_{2}, \ldots, v_{2 n}\right\}$

$E=\left\{v_{i} v_{i+1}: 1 \leq i \leq 2 n-1\right\} \cup\left\{v_{2 n} v_{1}\right\}$

Refer Figure 1(a) and 2(a),

Now let us label the vertices of $C_{2 n}$ as follows:

Case 1: $(2 n-1)$ is prime.

$f\left(v_{i}\right)=k+i-1$, for $1 \leq i \leq 2 n$ and $k \not \equiv 0(\bmod (2 n-1))$.

It is easy to check that the induced function $f^{+}: E(G) \rightarrow N$ of edges defined by $f^{+}\left(v_{i} v_{i+1}\right)=\operatorname{gcd}\left(f\left(v_{i}\right), f\left(v_{i+1}\right)=1\right.$ and $f^{+}\left(v_{2 n} v_{1}\right)=\operatorname{gcd}\left(f\left(v_{2 n}\right), f\left(v_{1}\right)=1 \quad \forall e \in E\left(C_{2 n}\right)\right.$.

This is clear from Figure $1(b)$.

Case 2: $(2 n-1)$ is not prime.

$f\left(v_{i}\right)=k+i-1$, for $1 \leq i \leq 2 n$ and $k$ not a multiple factor of $(2 n-1))$.

It is easy to check that the induced function $f^{+}: E(G) \rightarrow N$ of edges defined by $f^{+}\left(v_{i} v_{i+1}\right)=\operatorname{gcd}\left(f\left(v_{i}\right), f\left(v_{i+1}\right)=1\right.$ and $f^{+}\left(v_{2 n} v_{1}\right)=\operatorname{gcd}\left(f\left(v_{2 n}\right), f\left(v_{1}\right)=1 \quad \forall e \in E\left(C_{2 n}\right)\right.$.

This is clear from Figure $2(b)$. Hence $C_{2 n}$ admits $k$ - prime labeling. 


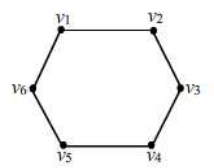

(a)

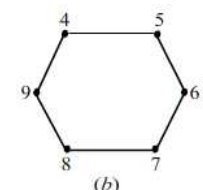

Figure 1. (a) $C_{6}$ (b) $k$-prime labeling of $C_{6}$ for $k=4$

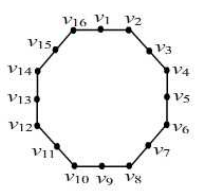

(a)

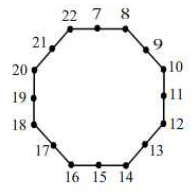

(b)
Figure 2. (a) $C_{16}$ (b) $k$-prime labeling of $C_{16}$ for $k=7$

Theorem 3.2. Odd Cycle $C_{2 n+1}$ is $k$ - prime for $k$ odd and $n=1,2,4$.

Proof. Let us denote the vertices and edges of $C_{2 n+1}$ as $V=\left\{v_{1}, v_{2}, \ldots, v_{2 n+1}\right\}$

$E=\left\{v_{i} v_{i+1}: 1 \leq i \leq 2 n\right\} \cup\left\{v_{2 n+1} v_{1}\right\}$

Refer Figure 3(a),

Now let us label the vertices of $\mathrm{C}_{2 n+1}$ as follows:

$f\left(v_{i}\right)=k+i-1$, for $1 \leq i \leq 2 n+1$.

It is easy to check that the induced function $f^{+}: E(G) \rightarrow N$ of edges defined by $f^{+}\left(v_{i} v_{i+1}\right)=\operatorname{gcd}\left(f\left(v_{i}\right), f\left(v_{i+1}\right)=1\right.$ and $f^{+}\left(v_{2 n+1} v_{1}\right)=\operatorname{gcd}\left(f\left(v_{2 n+1}\right), f\left(v_{1}\right)=1 \quad \forall e \in E\left(C_{2 n+1}\right)\right.$.

This is clear from Figure 3(b).

Hence $\mathrm{C}_{2 n+1}$ admits $k$ - prime labeling.
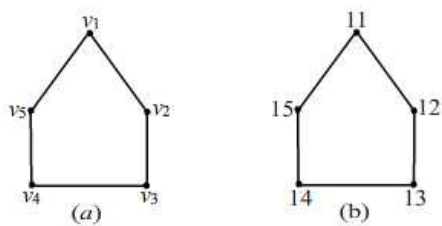

Figure 3. (a) $C_{5}$ (b) $k$ - prime labeling of $C_{5}$ for $k=11$

Theorem 3.3. Tadpole graph $T_{n, m}$ is $k$-prime for all even positive integer $n \geq 4$ and $m \geq 1$.

Proof. Let us denote the vertices and edges of $T_{n, m}$ as

$V=\left\{v_{1}, v_{2}, \ldots, v_{n+m}\right\}$

$E=\left\{v_{i} v_{i+1}: 1 \leq i \leq n+m-1\right\} \cup\left\{v_{n} v_{1}\right\}$

Refer Figure 4(a),

Now let us label the vertices of $T_{n, m}$ as follows:

Case 1: $(n-1)$ is prime.

$f\left(v_{i}\right)=k+i-1$, for $1 \leq i \leq n+m$ and $k \not \equiv 0(\bmod (n-1))$.

It is easy to check that the induced function $f^{+}: E(G) \rightarrow N$

of edges defined by $f^{+}\left(v_{i} v_{i+1}\right)=\operatorname{gcd}\left(f\left(v_{i}\right), f\left(v_{i+1}\right)=1\right.$ and $f^{+}\left(v_{n} v_{1}\right)=\operatorname{gcd}\left(f\left(v_{n}\right), f\left(v_{1}\right)=1 \quad \forall e \in E\left(T_{n, m}\right)\right.$.

Case 2: $(n-1)$ is not prime.

$f\left(v_{i}\right)=k+i-1$, for $1 \leq i \leq n+m$ and $k$ not a multiple factor of $(n-1))$.
It is easy to check that the induced function $f^{+}: E(G) \rightarrow N$ of edges defined by $f^{+}\left(v_{i} v_{i+1}\right)=\operatorname{gcd}\left(f\left(v_{i}\right), f\left(v_{i+1}\right)=1\right.$ and $f^{+}\left(v_{n} v_{1}\right)=\operatorname{gcd}\left(f\left(v_{n}\right), f\left(v_{1}\right)=1 \quad \forall e \in E\left(T_{n, m}\right)\right.$

This is clear from Figure $4(b)$.

Hence $T_{n, m}$ admits $k$ - prime labeling.

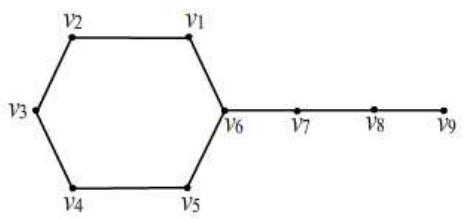

(a)

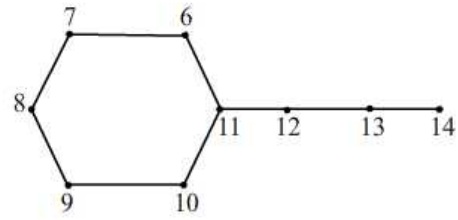

(b)

Figure 4. (a) $T_{6,3}$ (b) $k$ - prime labeling of $T_{6,3}$ for $k=6$

Theorem 3.4. Tadpole graph $T_{n, m}$ is $k$ - prime for $k$ odd, $n=3,5,9$ and $m \geq 1$.

Proof. Let us denote the vertices and edges of $T_{n, m}$ as

$V=\left\{v_{1}, v_{2}, \ldots, v_{n+m}\right\}$

$E=\left\{v_{i} v_{i+1}: 1 \leq i \leq n+m-1\right\} \cup\left\{v_{n} v_{1}\right\}$

Refer Figure 5(a),

Now let us label the vertices of $T_{n, m}$ as follows:

$f\left(v_{i}\right)=k+i-1$, for $1 \leq i \leq n+m$.

It is easy to check that the induced function $f^{+}: E(G) \rightarrow N$ of edges defined by $f^{+}\left(v_{i} v_{i+1}\right)=\operatorname{gcd}\left(f\left(v_{i}\right), f\left(v_{i+1}\right)=1\right.$ and $f^{+}\left(v_{n} v_{1}\right)=\operatorname{gcd}\left(f\left(v_{n}\right), f\left(v_{1}\right)=1 \quad \forall e \in E\left(T_{n, m}\right)\right.$

This is clear from Figure $5(b)$.

Hence $T_{n, m}$ admits $k$ - prime labeling.

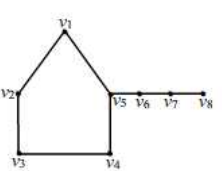

(a)

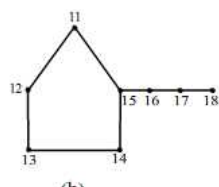

(b)
Figure 5. (a) $T_{5,3}$ (b) $k$ - prime labeling of $T_{5,3}$ for $k=11$

Theorem 3.5. Friendship graph $F_{n}(n \geq 3)$ is $k$ - prime for $k$ odd.

Proof. Let us denote the vertices and edges of $F_{n}$ as

$V=\left\{v, v_{1}, v_{2}, \ldots, v_{2 n}\right\}$

$E=\left\{v v_{i}: 1 \leq i \leq 2 n\right\} \cup\left\{v_{i} v_{i+1}: i=1,3,5, \ldots, 2 n-1\right\}$

Refer Figure 6(a), 
Now let us label the vertices of $F_{n}$ as follows:

$f(v)=p$, where $p$ is largest prime less than or equal to $k+2 n$ and remaining $2 n$ vertices are distinctly labeled from $\{k, k+$ $1, \ldots, k+2 n\}-p$.

$f\left(v_{i}\right)=k+i-1$, for $1 \leq i \leq 2 n$.

It is easy to check that the induced function $f^{+}: E(G) \rightarrow N$ of edges defined by $f^{+}\left(v v_{i}\right)=\operatorname{gcd}\left(f(v), f\left(v_{i}\right)=1\right.$ and $f^{+}\left(v_{i} v_{i+1}\right)=\operatorname{gcd}\left(f\left(v_{i}\right), f\left(v_{i+1}\right)\right)=1 \quad \forall e \in E\left(F_{n}\right)$.

This is clear from Figure $6(b)$.

Hence $F_{n}$ admits $k$ - prime labeling.

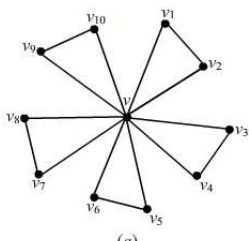

(a)

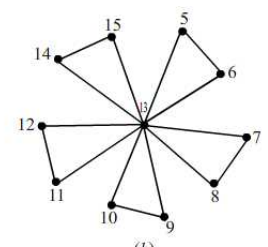

(b)
Figure 6. (a) $F_{5}$ (b) $k$-prime labeling of $F_{5}$ for $k=5$

Theorem 3.6. The barycentric subdivision $C_{n}\left(C_{n}\right)$ of cycle $C_{n}$ is $k$-prime for $n \geq 3$.

Proof. Case 1: $(2 n-1)$ is prime.

Sub Case $(a)$ : When $k$ is odd

Let $u_{1}, u_{2}, \ldots, u_{n}$ be the vertices of the cycle $C_{n}$. Let $v_{1}, v_{2}, \ldots, v_{n}$ be the newly inserted vertices of $C_{n}\left(C_{n}\right)$ where $v_{i+1}$ subdivides the edge $u_{i} u_{i+1}$ for $1 \leq i \leq n-1$ and $v_{1}$ subdivides the edge $u_{n} u_{1}$.

Now let us label the vertices of $C_{n}\left(C_{n}\right)$ as follows:

Let us denote the vertices and edges of $C_{n}\left(C_{n}\right)$ as

$V=\left\{u_{1}, u_{2}, \ldots, u_{n}\right\} \cup\left\{v_{1}, v_{2}, \ldots, v_{n}\right\}$

$E=\left\{v_{i} u_{i}: 1 \leq i \leq n\right\} \cup\left\{u_{i} v_{i+1}: 1 \leq i \leq n-1\right\} \cup\left\{u_{n} v_{1}\right\} \cup$

$\left\{v_{i} v_{i+1}: 1 \leq i \leq n-1\right\} \cup\left\{v_{n} v_{1}\right\}$

Refer Figure 7(a),

Now let us label the vertices of $C_{n}\left(C_{n}\right)$ as follows:

$f\left(v_{1}\right)=k$

$f\left(u_{1}\right)=k+1$

$f\left(v_{i}\right)=k+2(i-1), 2 \leq i \leq n$,

$f\left(u_{i}\right)=k+2 i-1,2 \leq i \leq n$,

It is easy to check that the induced function $f^{+}: E(G) \rightarrow N$ of edges defined by $f^{+}\left(v_{i} u_{i}\right)=\operatorname{gcd}\left(f\left(v_{i}\right), f\left(u_{i}\right)\right)=1$

$f^{+}\left(u_{i} v_{i+1}\right)=\operatorname{gcd}\left(f\left(u_{i}\right), f\left(v_{i+1}\right)\right)=1$

$f^{+}\left(u_{n} v_{1}\right)=\operatorname{gcd}\left(f\left(u_{n}\right), f\left(v_{1}\right)\right)=1$

$f^{+}\left(v_{i} v_{i+1}\right)=\operatorname{gcd}\left(f\left(v_{i}\right), f\left(v_{i+1}\right)\right)=1$ and

$f^{+}\left(v_{n} v_{1}\right)=\operatorname{gcd}\left(f\left(v_{n}\right), f\left(v_{1}\right)\right)=1 \quad \forall e \in E\left(C_{n}\left(C_{n}\right)\right)$.

This is clear from Figure 7(b).

Sub Case $(b)$ : When $k$ is even

Let $u_{1}, u_{2}, \ldots, u_{n}$ be the vertices of the cycle $C_{n}$. Let $v_{1}, v_{2}, \ldots, v_{n}$ be the newly inserted vertices of $C_{n}\left(C_{n}\right)$ where $v_{i}$ subdivides the edge $u_{i} u_{i+1}$ for $1 \leq i \leq n-1$ and $v_{n}$ subdivides the edge $u_{n} v_{1}$.

Now let us label the vertices of $C_{n}\left(C_{n}\right)$ as follows:

Let us denote the vertices and edges of $C_{n}\left(C_{n}\right)$ as
$V=\left\{u_{1}, u_{2}, \ldots, u_{n}\right\} \cup\left\{v_{1}, v_{2}, \ldots, v_{n}\right\}$

$E=\left\{u_{i} v_{i}: 1 \leq i \leq n\right\} \cup\left\{v_{i} u_{i+1}: 1 \leq i \leq n-1\right\} \cup\left\{v_{n} u_{1}\right\} \cup$

$\left\{v_{i} v_{i+1}: 1 \leq i \leq n-1\right\} \cup\left\{v_{n} v_{1}\right\}$

Refer Figure 8(a),

Let us label the vertices of $C_{n}\left(C_{n}\right)$ as follows:

$f\left(u_{1}\right)=k$

$f\left(v_{1}\right)=k+1$

$f\left(u_{i}\right)=k+2(i-1), 2 \leq i \leq n$,

$f\left(v_{i}\right)=k+2 i-1,2 \leq i \leq n$,

It is easy to check that the induced function $f^{+}: E(G) \rightarrow N$

of edges defined by $f^{+}\left(u_{i} v_{i}\right)=\operatorname{gcd}\left(f\left(u_{i}\right), f\left(v_{i}\right)\right)=1$

$f^{+}\left(v_{i} u_{i+1}\right)=\operatorname{gcd}\left(f\left(v_{i}\right), f\left(u_{i+1}\right)\right)=1$

$f^{+}\left(v_{n} u_{1}\right)=\operatorname{gcd}\left(f\left(v_{n}\right), f\left(u_{1}\right)\right)=1$

$f^{+}\left(v_{i} v_{i+1}\right)=\operatorname{gcd}\left(f\left(v_{i}\right), f\left(v_{i+1}\right)\right)=1$ and

$f^{+}\left(v_{n} v_{1}\right)=\operatorname{gcd}\left(f\left(v_{n}\right), f\left(v_{1}\right)\right)=1 \quad \forall e \in E\left(C_{n}\left(C_{n}\right)\right)$.

This is clear from Figure $8(b)$.

Case 2: $(2 n-1)$ is not prime.

Sub Case (a): When $k$ is odd

Let $u_{1}, u_{2}, \ldots, u_{n}$ be the vertices of the cycle $C_{n}$. Let $v_{1}, v_{2}, \ldots, v_{n}$ be the newly inserted vertices of $C_{n}\left(C_{n}\right)$ where $v_{i+1}$ subdivides the edge $u_{i} u_{i+1}$ for $1 \leq i \leq n-1$ and $v_{1}$ subdivides the edge $u_{n} u_{1}$.

Now let us label the vertices of $C_{n}\left(C_{n}\right)$ as follows:

Let us denote the vertices and edges of $C_{n}\left(C_{n}\right)$ as

$V=\left\{u_{1}, u_{2}, \ldots, u_{n}\right\} \cup\left\{v_{1}, v_{2}, \ldots, v_{n}\right\}$

$E=\left\{v_{i} u_{i}: 1 \leq i \leq n\right\} \cup\left\{u_{i} v_{i+1}: 1 \leq i \leq n-1\right\} \cup\left\{u_{n} v_{1}\right\} \cup$

$\left\{v_{i} v_{i+1}: 1 \leq i \leq n-1\right\} \cup\left\{v_{n} v_{1}\right\}$

Refer Figure 9(a),

Now let us label the vertices of $C_{n}\left(C_{n}\right)$ as follows:

$f\left(v_{1}\right)=k$

$f\left(u_{1}\right)=k+1$

$f\left(v_{i}\right)=k+2(i-1), 2 \leq i \leq n$,

$f\left(u_{i}\right)=k+2 i-1,2 \leq i \leq n$,

It is easy to check that the induced function $f^{+}: E(G) \rightarrow N$

of edges defined by $f^{+}\left(v_{i} u_{i}\right)=\operatorname{gcd}\left(f\left(v_{i}\right), f\left(u_{i}\right)\right)=1$

$f^{+}\left(u_{i} v_{i+1}\right)=\operatorname{gcd}\left(f\left(u_{i}\right), f\left(v_{i+1}\right)\right)=1$

$f^{+}\left(u_{n} v_{1}\right)=\operatorname{gcd}\left(f\left(u_{n}\right), f\left(v_{1}\right)\right)=1$

$f^{+}\left(v_{i} v_{i+1}\right)=\operatorname{gcd}\left(f\left(v_{i}\right), f\left(v_{i+1}\right)\right)=1$ and

$f^{+}\left(v_{n} v_{1}\right)=\operatorname{gcd}\left(f\left(v_{n}\right), f\left(v_{1}\right)\right)=1 \quad \forall e \in E\left(C_{n}\left(C_{n}\right)\right)$.

This is clear from Figure $9(b)$.

Sub Case (b): When $k$ is even

Let $u_{1}, u_{2}, \ldots, u_{n}$ be the vertices of the cycle $C_{n}$. Let $v_{1}, v_{2}, \ldots, v_{n}$ be the newly inserted vertices of $C_{n}\left(C_{n}\right)$ where $v_{i}$ subdivides the edge $u_{i} u_{i+1}$ for $1 \leq i \leq n-1$ and $v_{n}$ subdivides the edge $u_{n} v_{1}$.

Now let us label the vertices of $C_{n}\left(C_{n}\right)$ as follows:

Let us denote the vertices and edges of $C_{n}\left(C_{n}\right)$ as

$V=\left\{u_{1}, u_{2}, \ldots, u_{n}\right\} \cup\left\{v_{1}, v_{2}, \ldots, v_{n}\right\}$

$E=\left\{u_{i} v_{i}: 1 \leq i \leq n\right\} \cup\left\{v_{i} u_{i+1}: 1 \leq i \leq n-1\right\} \cup\left\{v_{n} u_{1}\right\} \cup$

$\left\{v_{i} v_{i+1}: 1 \leq i \leq n-1\right\} \cup\left\{v_{n} v_{1}\right\}$

Refer Figure 10(a),

Let us label the vertices of $C_{n}\left(C_{n}\right)$ as follows:

$f\left(u_{1}\right)=k$

$f\left(v_{1}\right)=k+1$ 
$f\left(u_{i}\right)=k+2(i-1), 2 \leq i \leq n$,

$f\left(v_{i}\right)=k+2 i-1,2 \leq \bar{i} \leq n$,

It is easy to check that the induced function $f^{+}: E(G) \rightarrow N$ of edges defined by $f^{+}\left(u_{i} v_{i}\right)=\operatorname{gcd}\left(f\left(u_{i}\right), f\left(v_{i}\right)\right)=1$

$f^{+}\left(v_{i} u_{i+1}\right)=\operatorname{gcd}\left(f\left(v_{i}\right), f\left(u_{i+1}\right)\right)=1$

$f^{+}\left(v_{n} u_{1}\right)=\operatorname{gcd}\left(f\left(v_{n}\right), f\left(u_{1}\right)\right)=1$

$f^{+}\left(v_{i} v_{i+1}\right)=\operatorname{gcd}\left(f\left(v_{i}\right), f\left(v_{i+1}\right)\right)=1$ and

$f^{+}\left(v_{n} v_{1}\right)=\operatorname{gcd}\left(f\left(v_{n}\right), f\left(v_{1}\right)\right)=1 \quad \forall e \in E\left(C_{n}\left(C_{n}\right)\right)$.

This is clear from Figure 10(b).

Hence $C_{n}\left(C_{n}\right)$ admits $k$ - prime labeling.

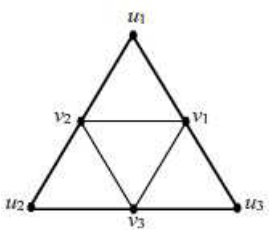

(a)

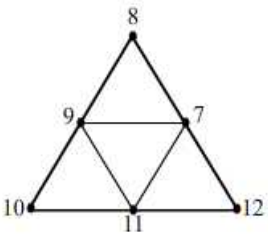

(b)
Figure 7. (a) $C_{3}\left(C_{3}\right)$ (b) $k$ - prime labeling of $C_{3}\left(C_{3}\right)$ for $k=$ 7

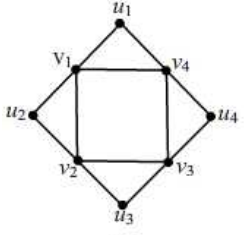

(a)

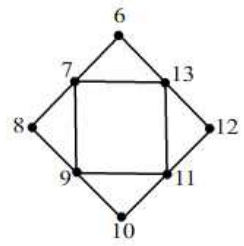

(b)

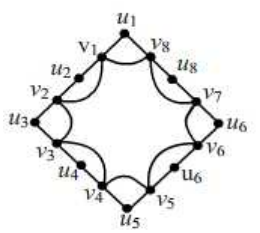

(a)

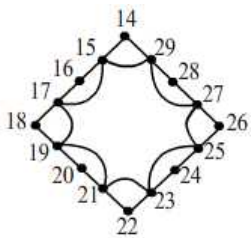

(b)
Figure 10. (a) $C_{8}\left(C_{8}\right)$ (b) $k$ - prime labeling of $C_{8}\left(C_{8}\right)$ for $k=$ 14

[2] S.K. Vaidya and U.M. Prajapati, Some Results on Prime and $k$ - Prime Labeling, Journal of Mathematics Research, 3(1)(2011)

$$
\begin{gathered}
\star \star \star \star \star \star \star \star \star \star ~ \\
\text { ISSN(P):2319-3786 }
\end{gathered}
$$

Malaya Journal of Matematik

$\operatorname{ISSN}(\mathrm{O}): 2321-5666$

Figure 8. (a) $C_{4}\left(C_{4}\right)$ (b) $k$ - prime labeling of $C_{4}\left(C_{4}\right)$ for $k=$ 6

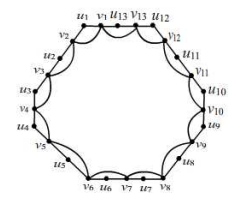

(a)

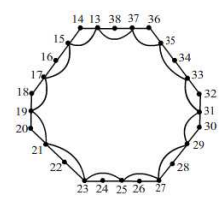

(b)

Figure 9. (a) $C_{13}\left(C_{13}\right)$ (b) $k$ - prime labeling of $C_{13}\left(C_{13}\right)$ for $k=13$

\section{Conclusion}

In this paper we have proved that Cycle graph $C_{n}$, Tadpole graph $T_{n, m}$, Friendship graph $F_{n}$ and Barycentric subdivision $C_{n}\left(C_{n}\right)$ is $k$ - prime. We have discussed $k$ - prime labeling of these graphs for any positive integer $k$. Further our study is extended to snake related graphs.

\section{References}

[1] A. Tout, A.N. Dabboucy and K. Howalla, Prime labeling of graphs, Nat. Acad. Sci letters, 11(1982), 365 - 368. 